\title{
Management of psychiatric complications in unrelated donor before unrelated peripheral hematopoietic stem cell collections
}

This article was published in the following Dove Press journal:

Journal of Blood Medicine

30 December 2016

Number of times this article has been viewed

\author{
Olivier Hequet ${ }^{1,2}$ \\ Valerie Mialou ${ }^{2}$ \\ Francoise Audat ${ }^{3}$ \\ Eric Wattel ${ }^{4}$ \\ Valerie Chapel ${ }^{4}$ \\ Damiela Revesz' \\ Jean-Piere Jouet ${ }^{3}$ \\ Brigitte Fisseaux ${ }^{5}$ \\ Mohamed Saoud ${ }^{6}$ \\ Mauricette Michallet ${ }^{4}$ \\ 'Apheresis Unit, ${ }^{2}$ Cell Therapy \\ Laboratory, Etablissement Français \\ du Sang (EFS) Rhône Alpes, Centre \\ Hospitalier Lyon Sud, Pierre Bénite, \\ ${ }^{3}$ Biomedicine Agency, Saint-Denis, \\ ${ }^{4}$ Hematological Unit, Hospices \\ Civils de Lyon, Centre Hospitalier \\ Lyon Sud, Pierre Bénite, ${ }^{5}$ Psychiatric \\ Unit, Hospices Civils de Lyon, \\ Centre Hospitalier, Bourgoin \\ Jallieu, ${ }^{6}$ Psychiatric Unit, Hospices \\ Civils de Lyon, Centre Hospitalier \\ PWertheimer, Lyon, France
}

\begin{abstract}
Allogeneic hematopoietic stem cell transplantation can efficiently treat patients with severe hematological diseases. A human leukocyte antigen-compatible donor is required for performing transplantation. The occurrence of unexpected acute severe diseases in a donor can compromise the feasibility of allogeneic hematopoietic stem cell transplantation. However, when a severe health problem occurs in a donor while the recipient has already received a conditioning regimen, hematologists have to find the best solutions for the recipient, while the team in charge of the donor has to find the best medical solutions for the donor. We describe here the occurrence of psychiatric acute complications in an unrelated donor while the myeloablative conditioning regimen had already been given to the recipient. We report the successive decisions that were made in an emergency based upon the expertise of physicians specialized in hematology, apheresis, cell therapy, and psychiatry to preserve the donor's health and recipient's life.

Keywords: hematopoietic stem cells, mobilization, harvest, psychiatric complication, CD34+ cells, unrelated donor
\end{abstract}

\section{Introduction}

Allogeneic hematopoietic stem cell transplantation (HSCT) is a therapeutic option to cure patients with severe hematological diseases. ${ }^{1}$ When a patient needs allogeneic HSCT, a human leukocyte antigen (HLA)-matched donor must be found. Such donor can be found first among the patient's family (HLA-sibling donor); if no donor is available, then a donor can be selected among the registered volunteer donors in the international donor registry (unrelated donor). ${ }^{1}$ Before transplantation, predonation consultations must be performed for all types of donors. ${ }^{2}$ These consultations are performed by a hematologist and an anesthetist in the case of bone marrow hematopoietic stem cell (HSC) collection or by an apheresis physician for a peripheral blood HSC collection (PBSC). The purpose of these consultations is to assess the risk of transmitted infectious diseases from the donor to the recipient, detect potential medical risks for the donor, and explain to the donor each stage of the donation process and potential adverse events. At the end of these consultations, the donor's agreement should be obtained. ${ }^{2}$

The occurrence of donor adverse events or complications after bone marrow or PBSC harvest has been extensively described. ${ }^{3-6}$ However, the occurrence of complications before HSC collections and in particular before PBSC harvest has only been poorly described. ${ }^{5}$ Before PBSC harvest, the injection of granulocyte-colony stimulating factor (G-CSF) often induces bone and muscular pains and can also induce fatigue or headaches. ${ }^{5-7}$ Such complications are well known and can be easily prevented or treated. Other
Centre de santé, Banque de cellules tissus, Etablissement Français du Sang (EFS), Pavillon Marcel Bérard IG, Centre Hospitalier Lyon Sud, Chemin du Grand Revoyet, 69495 Pierre Bénite, France

Tel +33478862273

Fax + 33478862297

Email olivier.hequet@efs.sante.fr 
complications rarely occur in the donor, but if a complication such as an infection appears before the recipient conditioning regimen has begun, the G-CSF injections and PBSC collection process are stopped. In contrast, if such complications occur after the recipient conditioning regimen has begun, the donor is treated and PBSC collection has to be performed. The recipient's life is threatened if massive chemotherapy or irradiation prescribed as conditioning regimen is not followed by stem cell infusion. Psychiatric complications in the donor can occur but are often linked to the donation process and never require medical prescription or hospitalization. We describe here the serious psychiatric complications subsequent to the donation process itself from an unrelated donor at the very beginning of the recipient myeloablative conditioning regimen. Potential replacement solutions at that particular moment of the process are discussed.

\section{Case report}

A 35-year-old French male unrelated donor 10/10 was selected to perform a PBSC donation for allogeneic HSCT to a European patient. The donor gave his consent for the stem cell collection and for the analysis of the clinical features and biological parameters involved in this cell collection. Less than 30 days before the collection procedure by apheresis, the donor was evaluated during a preapheresis consultation by both the hematologist and apheresis physician. The patient was declared able to receive the injection of G-CSF and undergo the apheresis process. He had no significant medical history and showed no previous psychiatric episodes. The donor understood the stages of the PBSC donation, agreed on the medications (subcutaneous injections of G-CSF) and apheresis sessions, and signed the agreement.

As usual, the day before the injection, the nurse in charge of the allogeneic HSCT coordination phoned the donor to ensure that there were no clinical or logistical problems. Two days after beginning of the G-CSF injections (ie, 2 days before the apheresis sessions), the donor's sister called the apheresis center explaining that the donor felt very tired and had developed psychiatric complications such as hearing voices. The medical staff decided to hospitalize the donor in emergency for a psychiatric evaluation in order to treat him, appreciate whether he still had his discernment, and know whether he still agreed on continuing the PBSC donation process. The practitioner in charge of unrelated donors in the French donor registry was informed.

After visiting the donor, the first psychiatrist concluded that the patient had occurrence of a strong depression and absence of psychosis and treated him with antidepressive and sedative oral medications. The psychiatrist concluded that the donor still had his discernment, that the PBSC harvesting could continue during a short hospitalization in a hematological unit, and that a hospitalization in a psychiatric unit was necessary after PBSC harvesting. The donor still agreed to perform the PBSC harvesting. In parallel, the donor informed us that he had a brother who had been followed up for acute myeloid leukemia and treated by allogeneic HSCT and who unfortunately died from acute graft-versus-host disease 1 year before. At that moment, ie, 2 days before the apheresis, the G-CSF injections were still given. The physician in charge of the French allogeneic donor's registry informed the European hematological team in charge of the recipient. Besides this, the recipient had already received the complete myeloablative regimen.

The day after, ie, the day before the planned PBSC collection, a second psychiatrist examined the patient and concluded that the patient was experiencing a psychotic syndrome. The psychiatrist decided to hospitalize the donor in a psychiatric unit, under enforcement. All these decisions led to the withdrawal of the PBSC donation process and G-CSF injection. The apheresis and hematological staff worked together to ask for further complementary psychiatric expertise. At the same time, the staff questioned the ethical committee who concluded that the donation could not proceed further without the donor's discernment. As the recipient had received the entire myeloablative conditioning regimen, the registry staff looked for HLA-compatible 4/6 or $5 / 6$ cord blood (CB) units in emergency.

The day after, the donor was evaluated by a third psychiatrist independent from the apheresis, hematological, and registry staff. The psychiatrist concluded that the donor was going through a depression without evidence for a psychosis and that he still had his discernment and still agreed on performing the PBSC donation for the unrelated donor. The medical enforcement was removed, and the G-CSF injection was prescribed again.

The PBSC harvest started with a 1-day delay. A fourth psychiatrist and nurse specialized in psychiatry evaluated the donor just before, and were present during, the PBSC apheresis session. The psychiatric expert in allogeneic organ and cell donations confirmed the diagnosis of major depressive episode according to the Diagnostic and Statistical Manual of Mental Disorders, fourth edition, criteria without mood-incongruent delusions or hallucinations. These last two psychiatrists confirmed the need for antidepressive medication. Again, the donor signed an agreement stipulating that he agreed to perform one or two apheresis sessions. The apheresis sessions were performed by spectra device and were uneventful. An amount of $1.70 \times 10^{6} \mathrm{CD} 34+\mathrm{HSCs} / \mathrm{kg}$ (depending on the recipient's weight) was collected during the first apheresis session. The donor and psychiatric team in charge agreed to 
perform a second apheresis session, which permitted collection of $0.75 \times 10^{6} \mathrm{CD} 34+\mathrm{HSCs} / \mathrm{kg}$. No complication occurred during and after the first and second apheresis sessions.

A total of $2.45 \times 10^{6} \mathrm{CD} 34+/ \mathrm{kg}$ allogeneic PBSC was infused to the recipient with a 36-hour delay. The recipient engrafted within 25 days. In parallel, 3 weeks after beginning of the treatment, the donor went back home and worked normally.

\section{Discussion}

This case report describes the successful management of psychiatric disorder occurring before harvesting and, in particular, during mobilization of HSCs. Psychiatric complications before, during, and after PBSC are rare and consist of anxiety, fatigue, and anorexia. ${ }^{7}$ In our case, we asked the pharmacist company whether G-CSF could cause depressive or pseudopsychotic symptoms such as hearing voices. The company confirmed the absence of such side effects with G-CSF. The successive psychiatric evaluations concluded that the patient had depressive symptoms, absence of psychosis, and persistence of discernment, and the donor still agreed on donating. Fatigue and depressive symptoms were linked to the donor's private life.

The donor center, apheresis and hematological teams responsible for the donor, and the French Registry and hematologist in charge of the recipient were encouraged to find solutions in this very critical situation after the recipient had already received the myeloablative conditioning regimen. The strategy was to find $\mathrm{CB}$ units if the donation was not possible. In the same very short period of time, we asked for psychiatric expertise from a psychiatric expert in organ donations and psychiatrists in charge of exclusion criteria for the donation process. The psychiatric evaluations concluded it was possible to perform the PBSC with persistence of the donor's discernment. Moreover, for personal and psychological reasons, it was important for the donor to donate his HSCs, which reinforced the decision to perform the PBSC. The cytapheresis was performed with 1-day delay considering the fact that G-CSF was stopped during 1 day. This schedule was not optimal but enabled us to harvest a sufficient number of HSCs in only two apheresis sessions.

The retrospective analysis of the events that occurred for this donor led to the conclusion that no abnormality was caused by the decisions taken by the teams in charge of the donor. The donor had not mentioned his psychological and personal problems to any of the staff members in charge of the collection process. This reinforced the fact that a psychological evaluation should be performed on the donor to search for potential psychological, social, or private issues that could interfere with the donation. In our particular case, a psychological evaluation had not shown any contraindication. The successive evaluation had to be performed in a very short period of time, to keep or to reject the PBSC strategy, for the same donor, while a second solution from another donor or HSC source, such as CB units was being searched. This complication also illustrated the stress induced by the HSC donation. The donor had private issues, but the intensity of the psychological complications was maximal when beginning the G-CSF treatment. This raised the question of the psychological issues and motivations of allogeneic HSCT unrelated donors. ${ }^{3,4}$

This case report illustrates the complexity of the interactions encountered in allogeneic HSCT procedures. To prevent the occurrence of complications in both HSC donor and HSCT recipient or to timely and efficiently react in a coordinated manner, a global organization of the stages of action of the teams in charge should be defined according to the stages of mobilization, collection, transport, and HSCT. The teams in charge have to ensure the safety of the donor before and after HSC donation. ${ }^{8}$ For this purpose, nurses and hematologists qualified in allogeneic transplantation and doctors qualified in apheresis should check the various stages (contraindications for HSC donation, for mobilization using G-CSF, for apheresis) and safety of the donors at each stage. In case of a complication, these teams must be immediately able to manage the medical complications in the donor with the assistance of their local medical network. At the same time, the national team in charge of the coordination between the donor collection and HSC allogeneic transplantation should be informed. In practice, each country develops a national agency and subsequent departments themselves, coordinated to perform each HSC collection and allogeneic HSCT. All the departments and units involved in HSC collection and allogeneic HSCT should analyze the problem and help to find solutions together, considering the safety of the donor and recipient and preserving the ethical rules. The team in charge of the recipient should ensure allogeneic HSCT, including sufficient numbers of CD34+ cells as well as prophylactic and curative treatments of the complications. If the allogeneic HSCT cannot be performed, this team should find a replacement HSCT, for example by performing a CB allogeneic transplantation, and can be helped by the department in charge of the allogeneic HSCT coordination to find a CB HSC graft in emergency. The rules for good practices in HSC collection and transplantation are defined in each country. However, the guidelines given by accreditation organizations such as Joint Accreditation Committee can be considered as universal and applied by most of the teams who perform HSC collection and transplantation. ${ }^{9}$ Moreover, 
the different teams can be assisted by ethical committees to define the medical rules that must be followed in these particular situations.

\section{Conclusion}

While most of stem cell collections are performed in routine practice without occurrence of any complication, the knowledge of unusual complications allows anticipation of them during the procedure. For all complications, all teams in charge have to work in close collaboration to decide on the best solution to perform allogeneic HSCT in emergency situations while preserving the donor's health and recipient's life.

\section{Disclosure}

The authors report no conflicts of interest in this work.

\section{References}

1. Armitage JO. Bone marrow transplantation. $N$ Engl J Med. 1994; 330(12):827-838.
2. Horowitz MM, Confer DL. Evaluation of hematopoietic stem cell donors. Hematology Am Soc Hematol Educ Program. 2005:469-475.

3. Bredeson C, Leger C, Couban S, et al. An evaluation of the donor experience in the Canadian multicenter randomized trial of bone marrow versus peripheral blood allografting. Biol Blood Marrow Transplant. 2004;10(6):405-414.

4. Anderlini P, Donato M, Chan KW, et al. Allogeneic blood progenitor cell collection in normal donors after mobilization with filgastrim: the M.D. Anderson Cancer Center experience. Transfusion. 1999;39(6):555-560.

5. Miller JP, Perry EH, Price TH, et al. Recovery and safety profiles of marrow and PBSc donors: experience of the national marrow donor program. Biol Blood Marrow Transplant. 2008;14(9 Suppl):29-36.

6. Pulsipher MA, Chitphakdithai P, Miller JP, et al. Adverse events among 2408 unrelated donors of peripheral blood stem cells: results of a prospective trial from the National Marrow Donor Program. Blood. 2009;113(15):3604-3611.

7. Pulsipher MA, Chitphakdithai P, Logan BR, et al. Acute toxicities of unrelated bone marrow versus peripheral blood stem cell donation: results of a prospective trial from the National Marrow Donor Program. Blood. 2013;121(1):197-206.

8. Hequet $\mathrm{O}$. Hematopoietic stem and progenitor cell harvesting: technical advances and clinical utility. J Blood Med. 2015;6:55-67.

9. Caunday O, Bensoussan D, Decot V, Bordigoni P, Stoltz JF. Regulatory aspects of cellular therapy product in Europe: JACIE accreditation in a processing facility. Biomed Mater Eng. 2009;19(4-5):373-379.
Journal of Blood Medicine

\section{Publish your work in this journal}

The Journal of Blood Medicine is an international, peer-reviewed, open access, online journal publishing laboratory, experimental and clinical aspects of all aspect pertaining to blood based medicine including but not limited to: Transfusion Medicine; Blood collection, Donor issues, Transmittable diseases, and Blood banking logistics; Immunohematology; Artificial and alternative

\section{Dovepress}

blood based therapeutics; Hematology; Biotechnology/nanotechnology of blood related medicine; Legal aspects of blood medicine; Historical perspectives. The manuscript management system is completely online and includes a very quick and fair peer-review system. Visit http://www.dovepress.com/ testimonials.php to read real quotes from published authors. 